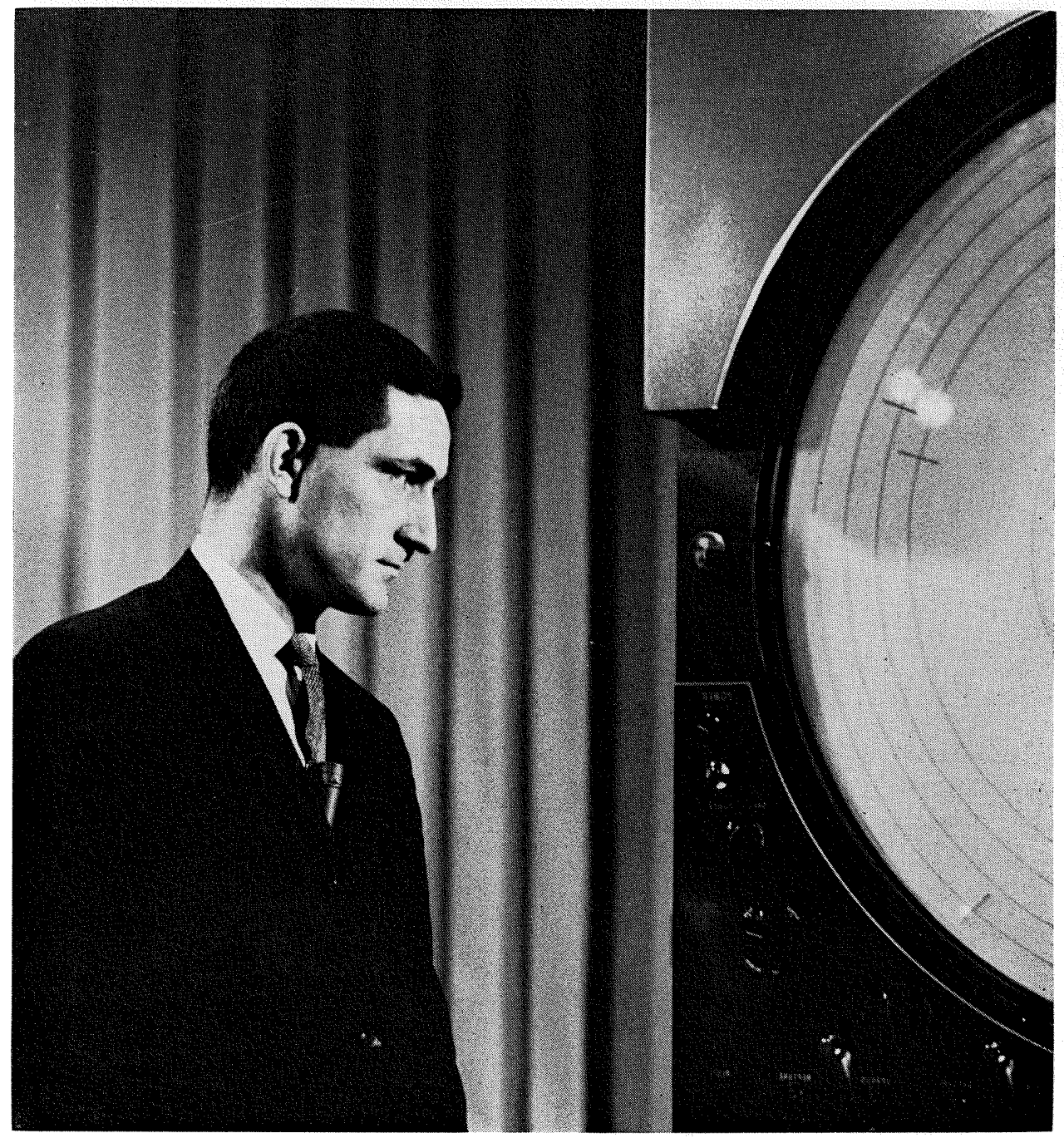

Albert R. Hibbs, director of the

Division of

Space Sciences

at Caltech's Jet

Propulsion

Laboratory.

by Albert R. Hibbs

\title{
The National Program for Lunar and Planetary Exploration
}

The Jet Propulsion Laboratory of the California Institute of Technology was established more than twenty years ago to undertake research and development of guided missiles. Until 1958, the end products of these activities were military weapons systems. For example, the Corporal - the nation's first operational guided missile - and the Sergeant - now entering operational status as a second-generation successor to the Corporal - were the products of research and development efforts of JPL. In the pursuit of its objectives, the Laboratory was responsible for num- erous fundamental developments in rocketry, guidance, communication, and instrumentation.

These skills, which the Laboratory had developed as the founder of American guided missile technology, were used in the design and construction of this country's first successful artificial satellite, Explorer I. At the time of launching of Explorer I, on January 31, 1958, the Laboratory was under contract to the United States Army, and it joined with its companion organization, the Army Ballistic Missile Agency (ABMA), under the technical leadership of Dr. Wern- 
her von Braun, in the launching of this satellite.

In addition to a series of successful launchings of artificial satellites, JPL and ABMA launched two probes intended for lunar exploration, Pioneer III and IV. Neither of these came sufficiently close to the moon to merit the label of lunar probe; nevertheless, Pioneer IV successfully escaped from the gravitational field of the earth to take up its independent orbit around the sun as this country's first artificial planet.

This successful launching occurred in March of 1959, three months after the Laboratory had been transferred from the direction of the United States Army to the direction of the National Aeronautics and Space Administration (NASA). For more than two years, the Jet Propulsion Laboratory has been one of the three space flight centers of the National Aeronautics and Space Administration.

The successful team which launched the Explorers and the first successful Pioneer has been kept intact. Dr. Wernher von Braun's group at Huntsville, Alabama, also has been transferred to the auspices of NASA and named the George C. Marshall Space Flight Center. The third center has been organized by NASA in Greenbelt, Maryland, under the name of the Goddard Space Flight Center.

The NASA has assigned to each of its three space flight centers their particular roles in the space flight program. Goddard Space Flight Center is constructing payloads for the earth-satellite and sounding-rocket program. The Marshall Space Flight Center in Huntsville, Alabama, is developing and operating the launching rockets. The Jet Propulsion Laboratory in Pasadena is developing the spacecraft for lunar and planetary exploration.

The lunar program is characterized by a steady increase in the complexity of exploring spacecraft. The program begins in 1961 with spacecraft development flights and continues in 1962 with "rough-landing" missions. Next, lunar orbiters, capable of photographing the moon from a few hundred kilometers

\section{JPL's Lunar Program}

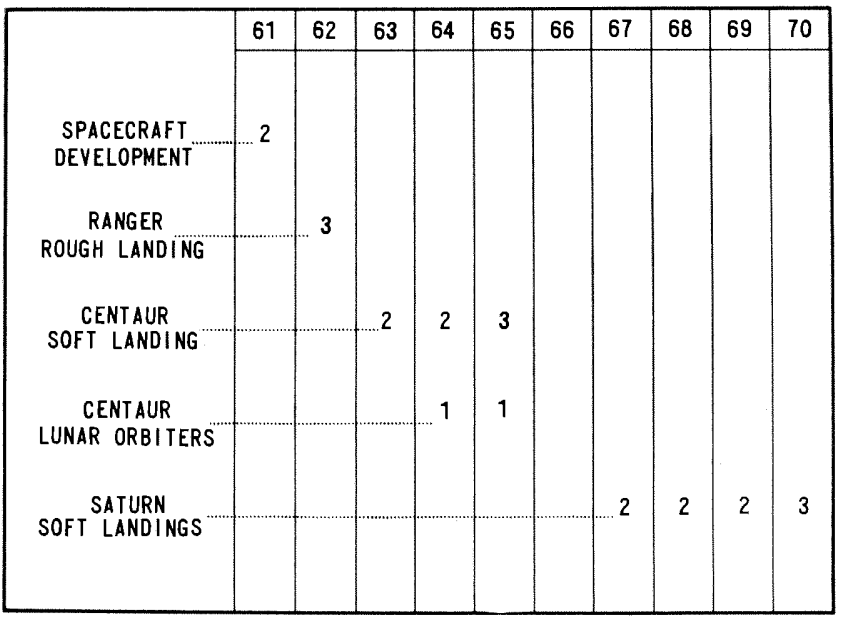

Flight Schedules to the Planets

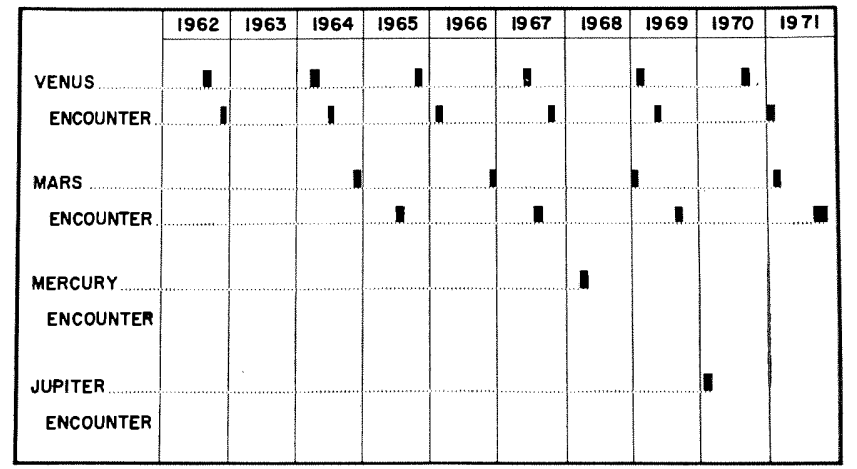

above it, and lunar soft-landers, capable of exploring their immediate environs, are scheduled in the period from 1963 to 1965.

In the second half of the decade, more complicated and larger lunar vehicles are employed. These vehicles are capable of returning samples of lunar material to the earth and roving over the surface to extend the exploration program to a much more diversified type of lunar material and surface.

The flight schedules to the planets are limited by the motions of the planets in their orbits. The planetary program will begin in 1962 with a Venus fly-by passing close enough to the planet to obtain better resolution in planetary measurements than could be obtained either from the earth or from an earth satellite in this same time period. The second flight, later in 1962, will gather only interplanetary data. Similar flights will be made past Venus in 1964 and possibly in 1965.

In 1963, a Mars spacecraft will be flight-tested and in 1964 will be flown on a mission to observe Mars.

Scientific-Experiment Plan: Ranger 1 and 2

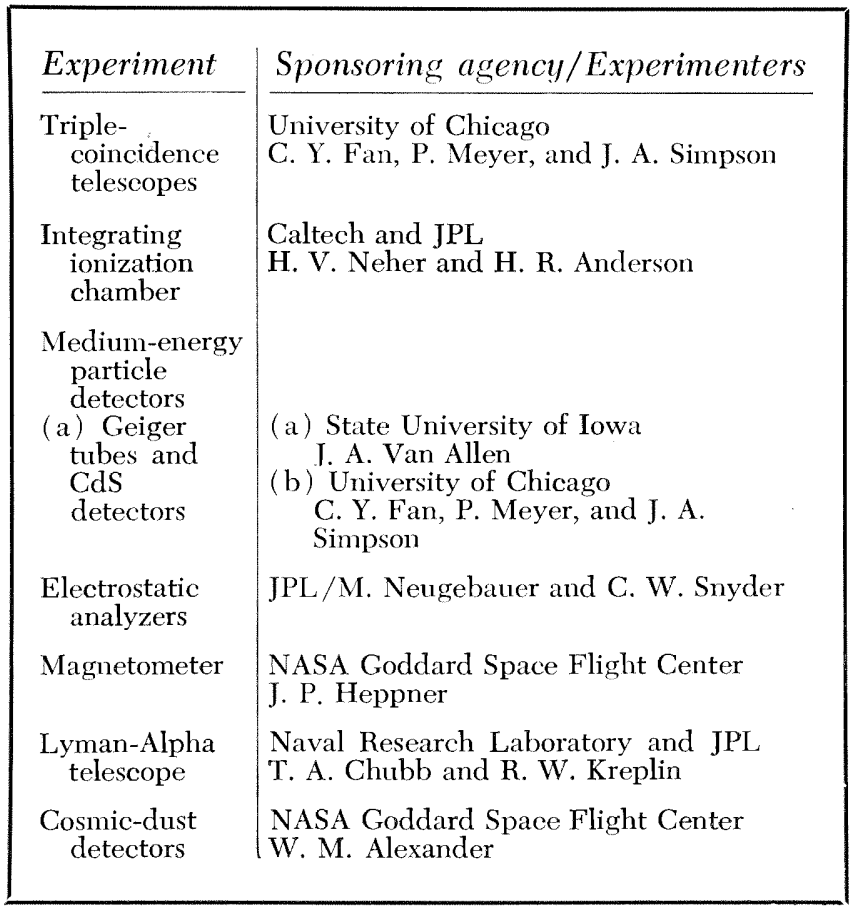


Scientific-Experiment Plan: Ranger 3, 4, 5

\begin{tabular}{|l|l|l|}
\hline Experiments & $\begin{array}{l}\text { Instruments and } \\
\text { measurements }\end{array}$ & $\begin{array}{l}\text { Cognizant agency } \\
\text { and scientist }\end{array}$ \\
\cline { 1 - 2 } $\begin{array}{l}\text { Capsule: } \\
\text { Seismology } \\
\text { seismometer } \\
\text { capsule temper- } \\
\text { ature measure- } \\
\text { ment }\end{array}$ & $\begin{array}{l}\text { Caltech/Columbia U. } \\
\text { F. Press/M. Ewing }\end{array}$ \\
$\begin{array}{l}\text { Bus: } \\
\text { Photography of } \\
\text { area } \\
\text { gammall lunar } \\
\text { spectroscopy }\end{array}$ & $\begin{array}{l}\text { vidicon television } \\
\text { gamma-ray } \\
\text { spectrometer }\end{array}$ & JPL/E. F. Dobies \\
& & $\begin{array}{l}\text { U of Calif./LASL/JPL } \\
\text { R. Arnold/M. A. } \\
\text { Van Dilla } \\
\text { E. C. Anderson/A. } \\
\text { Metzger }\end{array}$ \\
\hline
\end{tabular}

Availability of the Saturn booster in the second half of the decade will permit experiments with spacecraft put in orbit around the target planets and landed on the surface of Mars, and possibly on Venus, if continued temperature measurements indicate that this is practical.

The increased capability of Saturn vehicles will provide spacecraft flexibility in the exploitation of new discoveries and will allow such special missions as fly-bys of Mercury and Jupiter and a flight considerably out of the plane of the ecliptic to be flown near the end of the decade.

Only the very earliest spacecraft involved in the lunar and planetary programs can be described here since only for these is sufficient design detail available to make such a description valuable.

Two vehicles, Ranger 1 and 2, will be launched during the last half of 1961 to begin the interplanetary exploration program. The trajectories for these two spacecraft, and the trajectories for Ranger 3, 4, and 5 , are shown above.

Ranger 1 and 2 will be sent on a long elliptical trajectory whose apogee is approximately one million kilometers from the earth, which means that they will be launched with a speed only slightly less than escape speed. They will spend about one or two months measuring the characteristics of space at the order of several hundred thousand to a million kilometers from the earth.

Ranger 3, 4, and 5 will be sent on trajectories toward the moon and will carry with them a capsule containing a seismometer. This capsule will be detached from the main bus of the spacecraft, slowed by a retrorocket, and landed on the surface at a speed of a few hundred miles an hour.

Most of the scientific experiments which will be carried out by the first two Ranger spacecraft will be directed toward the objective of measuring interplanetary fields and charged particles. There are two exceptions to this category; namely, a measurement of density of interplanetary dust, and an observation of the neutral hydrogen geocorona.
The charged-particle measurements will be carried out by instruments covering a range of energies. At the lowest energy range, there are electrostatic analyzers capable of examining the spectrum of protons from 0 to 5000 electron volts and the spectrum of electrons up to a few hundred electron volts. These analyzers will thus be the first ones flown which extend their measurements into this very low region, characteristic of the hypothesized solar wind. Medium-range particles will be detected by a group of counters relying both on the solid-state property of semi-conductors and on traditional geiger tubes. Ionization chambers such as those flown on balloons in the earth's atmosphere, and triple-coincidence telescopes, such as those which were used on Pioneer V, will complete the charged-particle measurements by covering the highest energy range in the neighborhood of 10 to $100 \mathrm{mev}$ for protons.

Closely associated with the behavior of charged particles is, of course, the behavior of the interplanetary and magnetic field. This will be measured with a rubidium vapor magnetometer.

The neutral hydrogen cloud around the earth will be observed by a scanning telescope which detects scattered radiation of the Lyman-alpha frequency. As the spacecraft recedes from the earth, this telescope will repeatedly scan the vicinity of the earth, including in its successive pictures a larger and larger field of view.

The micrometeorite detectors on the Ranger will give information on both the energy and momentum of the particles striking it.

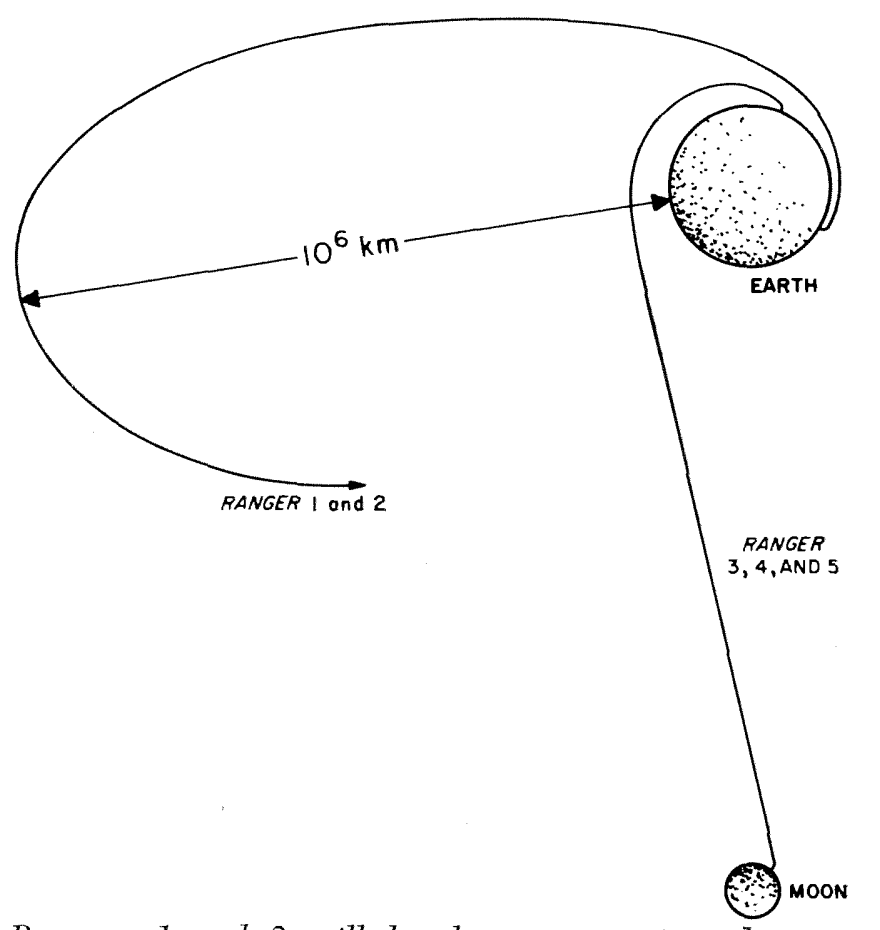

Rangers 1 and 2 will hurtle to approximately one million kilometers from the earth to explore interplanetary space. Rangers 3,4 and 5 take a path to the moon to drop seismometers on its surface. 
The model of the spacecraft for Rangers 1 and 2.

These probes will measure interplanetary fields and charged particles.

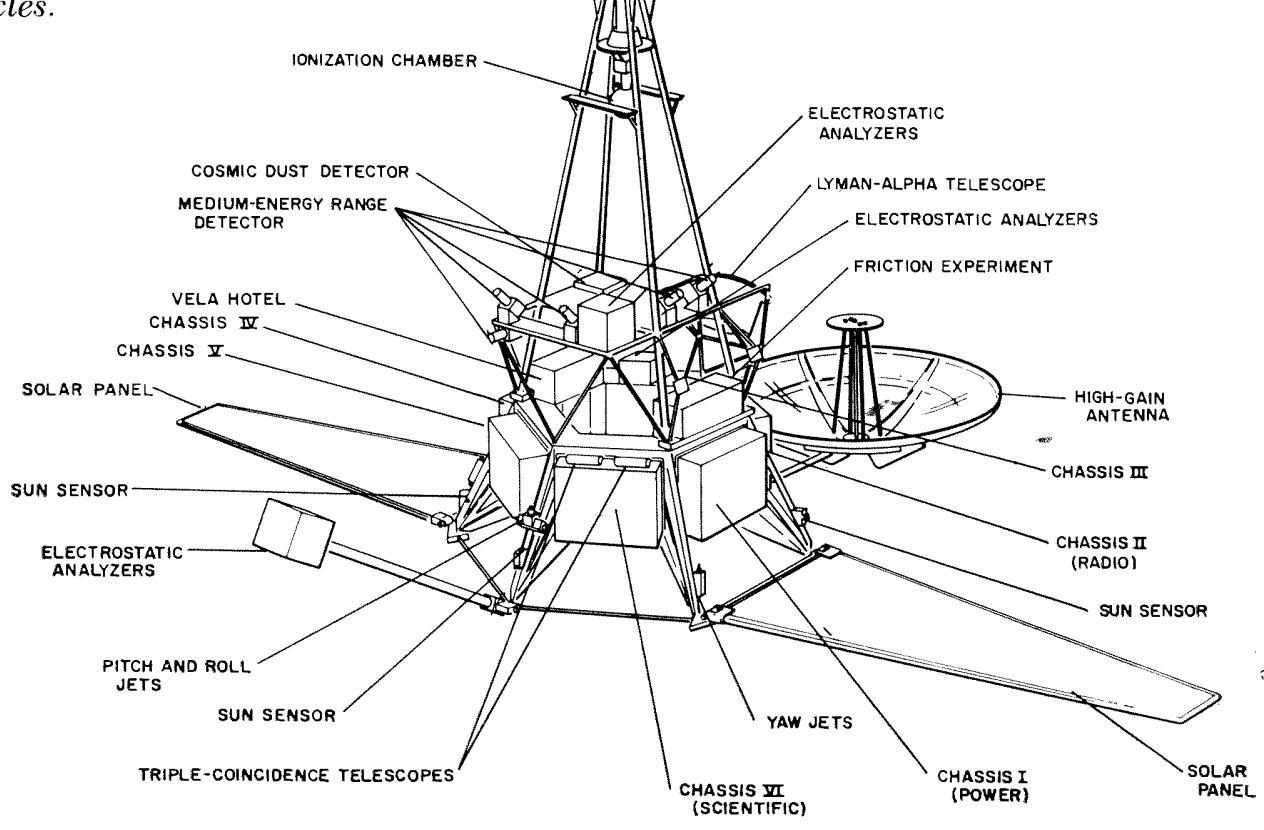

The spacecraft for Ranger 1 and 2 (above) has the rubidium vapor magnetometer located near the front end, where it is as far removed as possible from those parts of the spacecraft which may introduce a spurious magnetic field. The ionization chamber is below it, located in a position where it will be shielded as little as possible by the structure of the spacecraft. The six electrostatic analyzers are positioned so that they can see freely along opposite directions of each of three coordinate axes.

The spacecraft itself is powered by solar panels which operate after the attitude-control system has successfully aimed the spacecraft directly at the sun. The attitude-control system will thereafter maintain this aiming direction throughout the lifetime of the experiment. The directional parabolic antenna will be aimed at the earth with the same attitude-control system by means of rolling the spacecraft around its longitudinal axis after the sun direction has been fixed. In this way, and by hinging the antenna out from the spacecraft to the appropriate angle, the antenna can be made to point at the earth.

The Ranger 3, 4, and 5 spacecraft is similar in many ways to that used for Ranger 1 and 2. However, the superstructure containing the scientific instruments has been replaced by a superstructure supporting an omnidirectional antenna and surrounding the lunar capsule which together with its retromotor is being developed by the Aeronutronic Corporation under sub-contract to the Laboratory. This spacecraft is also powered by solar panels and communicates with the earth by means of a directional parabolic antenna.

As the spacecraft approaches the moon, a succession of photographs will be taken by a vidicon camera which is aided toward the lunar surface. The vidicon tube will employ a 200-line scan, and the optics will be such as to take a picture measuring approximately 40 kilometers on a side at the initiation of the picturetaking sequence and decreasing steadily to 600 meters on a side for the last picture expected to be successfully recovered from the data.

While the vidicon is in operation, a gamma-ray spectrometer, positioned far from the spacecraft so as to avoid the effect of secondaries, will measure the ambient radioactivity in the region of the spectral line associated with the decay of potassium 40. This experiment has been so designed that, even if the moon is composed of material as low in natural radioactivity as the chondritic meteorites, the detector will observe the lunar potassium 40 gamma rays above the background expected from interplanetary and cosmicray sources.

Approximately 30 kilometers above the lunar surface, after the spacecraft has been properly positioned, the capsule, together with its retromotor, will be detached from the parent spacecraft.

The capsule is spun to maintain its aiming direction. Thereafter, the retrorocket is ignited, which slows 
down the capsule to a zero speed relative to the lunar surface at an altitude of about 400 meters. The capsule then falls freely from this altitude to impact with a speed of about 30 meters per second.

Variations in retromotor performance will, of course, result in a variation of landing speeds. The expected standard deviation of landing speeds is approximately 30 to 40 meters per second. The landing capsule and all of the instrumentation within it - that is, the seismometer, its amplifier, transmitter, and antenna, power supply, righting mechanism, temperature control device, zeroing motor, and automatic calibration device - have all been designed to withstand several thousand G's of impact acceleration.

The seismometer carried in the capsule of Ranger 3,4 , and 5 is designed to operate thereafter for a period of 30 to 60 days. Even if no internal seismic activity occurs on the moon, it is likely that the impact of meteorites on the moon will create sufficient seismic disturbances to be detected by this device.

Detailed lists of experiments for the first flights in the planetary exploration program have not all been fully formalized. However, committees of the National Academy of Sciences' Space Science Board, as well as groups of consultants working with NASA personnel to advise the Space Science Steering Committee of NASA Headquarters, have considered in some detail the most pressing problems of planetary science which should be investigated with our earliest probes. A listing of experiments for the first Venus flight, the Mariner $A$, is now available.

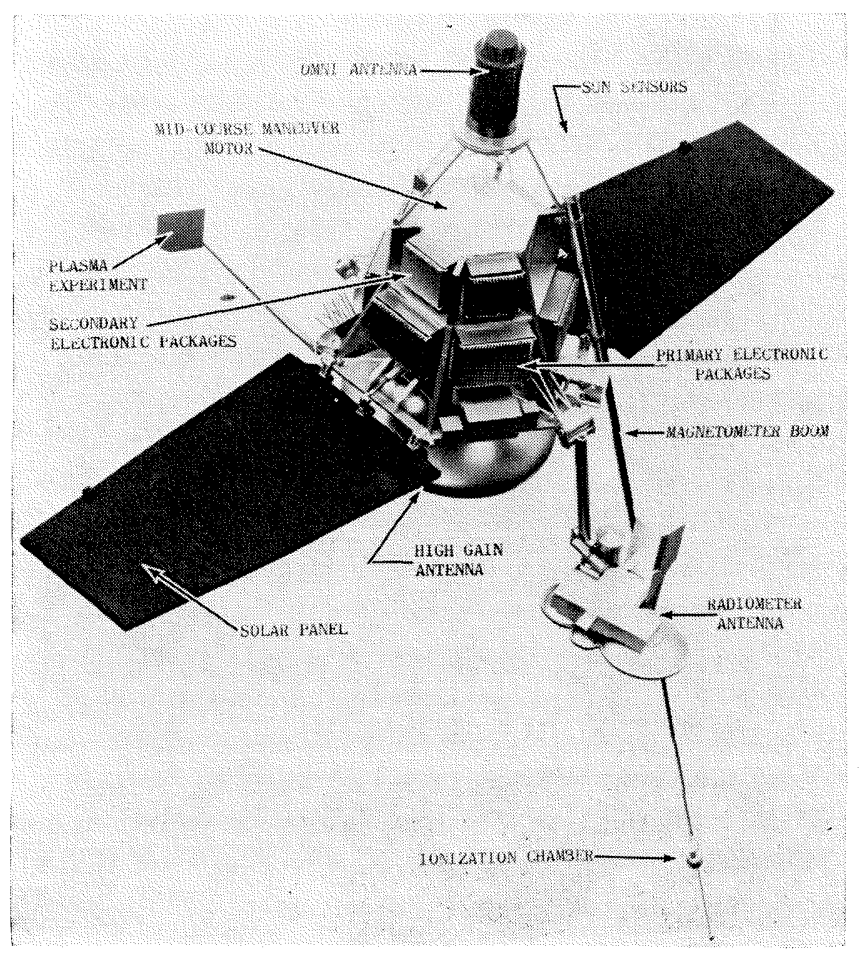

The Mariner A, designed to make the first exploratory flight to the near vicinity of Venus.
This spacecraft, designed to make a single pass in the near vicinity of Venus, will measure the temperature of the planet's surface, atmosphere, and ionosphere, evaluate the atmospheric composition of Venus; measure its magnetic field and the interplanetary magnetic field; and investigate the dust and chargedparticle spectrum near Venus and in interplanetary space. Some of the instruments for these experiments will produce their results only during the period of the near-pass of the planet. Others will operate all the way from earth to Venus. The magnetometer, for example, is in this latter class and consequently must be designed to measure not only the fields of the order of a few gammas which are expected to occur in interplanetary space, but also fields of the order of a gauss which may occur in the close vicinity of Venus.

A spacecraft designed to pass close to the planet Mars would carry very nearly the same instruments; however, their order of priority would differ. A difference which obviously affects the selection of instruments is the fact that the surface of Venus is not available to optical instruments, as is the surface of Mars.

Although the selection of experiments to be carried out by the first spacecraft to be soft-landed on the moon is not final a number of design studies of possible instruments have been completed. These studies have considered such devices as drills, geophysical instrument packages for both down-hole and surface measurements, and instruments for chemical and mineralogic analysis of surface material and material recovered from the drill hole.

In addition to these devices, the spacecraft will contain several television cameras with the total capability of a complete panorama sweep of the vicinity of the spacecraft as well as close-up observation of the material in the immediate neighborhood of the spacecraft. This Surveyor spacecraft is now being developed by the Hughes Aircraft Corporation under a subcontract with JPL.

The instrumentation and the spacecraft described here will initiate this nation's program for the exploration of the moon and planets. The successful development of this exploration program will yield, for example, geophysical and geochemical information about the moon which will help us to understand not only the nature of our sister planet but perhaps also something of the origin of the solar system, since the moon may still retain on its surface the five-billionyear-old record of these early processes. It is possible that the biologic exploration of Mars may reveal extraterrestrial life forms. The chemical analysis of such life, developing in an ecology completely separate from the earth, may bring us closer to the understanding of the origin of life. But even the excitement inherent in such possibilities as these will undoubtedly be surpassed by the reality of the discoveries which lie before us. 